\title{
O PROGRAMA SALF COMO FERRAMENTA PARA ANÁLISE DO PROCESSO DE LAMINAÇÃO A FRIO*
}

Gilberto Thiago de Paula Costa ${ }^{1}$ Carlos Augusto dos Santos ${ }^{2}$

\section{Resumo}

Este artigo apresenta o SALF, um software nacional desenvolvido para simular o processo de laminação a frio de chapas através do método dos elementos finitos. $O$ programa utiliza a formulação implícita/rígido-plástica em suas análises. Simulações retiradas da literatura foram realizadas com o software, sendo os materiais simulados alumínio e aço, com espessuras de 3,0 mm e $1,0 \mathrm{~mm}$, reduções de $5 \%$ e $30 \%$ e atrito de 0,06 a 0,15 . Os resultados do SALF ficaram bem próximos dos resultados descritos na literatura. Desta forma, a partir de agora, as siderúrgicas nacionais têm uma ferramenta nacional para simular o processo de laminação a frio, a qual pode auxiliar na melhoria de seus processos e produtos.

Palavras-chave: Laminação a frio de chapas; Método dos elementos finitos; Formulação implícita e rígido-plástica.

\section{SALF PROGRAM AS TOOL TO ANALYZE COLD FLAT ROLLING PROCESS}

\begin{abstract}
The article presents SALF program, a national software developed to simulate the cold rolling metal sheet process by the finite element method. The program uses the implicit and rigid-plastic approaches to perform numerical analyses. Simulations from the literature were performed using the program on materials such as steel and aluminum, with thicknesses of $1.0 \mathrm{~mm}$ and $3.0 \mathrm{~mm}$, reductions of $5 \%$ and $30 \%$, and friction coefficients of 0.1 and 0.3 . There was a strong approximation between the program results and those described in the simulations chosen from the literature. However, starting now domestic steel industries may use a national program to simulate the cold flat rolling process to improve their processes and products.

Keywords: Cold rolling of sheets; Finite element method; Implicit and rigid-plastic approaches.
\end{abstract}

1 Engenheiro Mecânico, Mestre em Montagem Industrial pela Universidade Federal Fluminense, Tecnologista, Divisão de Rejeitos Radiativos, Comissão Nacional de Energia Nuclear, Rio de Janeiro, Rio de Janeiro, Brasil.

2 Engenheiro Mecânico, Doutor em Engenharia Metalúrgica e de Minas pela Universidade Federal de Minas Gerais, Professor Adjunto IV, Departamento de Engenharia Mecânica, Escola de Engenharia, Universidade Federal Fluminense, Niterói, Rio de Janeiro, Brasil. 


\section{INTRODUÇÃO}

Dentro do universo dos processos de conformação mecânica, a laminação a frio desempenha um papel importante, uma vez que fornece matéria prima para uma série de produtos dos segmentos produtivos. Entre os segmentos que utilizam as chapas laminadas a frio pode-se ressaltar o setor de eletrodomésticos (chapas para fogões e geladeiras), a indústria da eletrônica (chapas para gabinetes de desktops e notebooks), a indústria do transporte (chapas para carroceria e chassis de carros, caminhões, ônibus). Além disso, é oportuno destacar o volume de aço laminado a frio atingiu o montante de 2 milhões de toneladas nos três primeiros trimestres de 2015 [1]. Dessa forma, considerando as colocações acima, fica evidente o papel importante da laminação a frio dentro da economia.

Para o estudo da laminação a frio, comumente são empregados três métodos, a saber: analítico, numérico e experimental. Os métodos analíticos [2-6] têm por característica básica a limitação em termos de não linearidade geométrica e de material, sendo na maioria das vezes utilizado para obter uma primeira aproximação da carga de laminação. Esses métodos datam de meados do século $X X$ e, ao longo do tempo, não tem apresentado evoluções. O estudo numérico tem sido realizado através do método dos elementos finitos (MEF) [7-13], o qual tem por característica principal fornecer informações sobre um grande número de variáveis do processo em um curto espaço de tempo. O MEF tem sido objeto de constantes modificações, as quais tem contribuído para sua melhor performance dentro das simulações de conformação mecânica. Os trabalhos experimentais [14-17] representam a única ferramenta a fornecer dados reais sobre o processo de laminação, servindo esses dados de referência para validação dos resultados do MEF. Os maiores avanços na área experimental tem sido o desenvolvimento de instrumentos de medições para auxiliar a leitura das variáveis do processo.

Atualmente, percebe-se que os métodos analíticos, quando adotados, continuam servindo como uma primeira aproximação da carga de laminação. O MEF juntamente com os estudos experimentais tem sido as abordagens mais utilizadas nos estudos da laminação a frio, devidos as vantagens mencionadas no parágrafo anterior. Infelizmente, em termos de MEF, as siderurgias nacionais ainda dependem da utilização de programas internacionais (ABAQUS, DEFORM), para simularem seus processos de laminação a frio. O custo de aquisição das licenças dos programas estrangeiros é elevado e também a assistência técnica fornecida no Brasil na maioria das vezes não responde adequadamente às necessidades das siderurgias. Dessa forma, mais uma vez as universidades são convocadas a desenvolver tecnologia para alimentar as indústrias nacionais, no caso em questão tal tecnologia se resume em um programa de MEF capaz de simular o processo de laminação a frio.

\section{OBJETIVO}

Diante do contexto apresentado na Introdução, este trabalho teve por objetivo principal o desenvolvimento de um programa para simular a operação de laminação de chapas a frio através do método dos elementos finitos. Em termos de objetivo secundário a intenção é o desenvolvimento de parcerias com as siderurgias nacionais visando a simulação de seus processos de laminação a frio através do software obtido. 
É importante destacar o caráter pioneiro do objetivo proposto neste trabalho, uma vez que o programa obtido com a pesquisa representa a primeira ferramenta nacional para simulação da laminação a frio de chapas via MEF.

\section{METODOLOGIA}

Visando à concretização do objetivo do trabalho, a metodologia foi dividida em cinco etapas: desenvolvimento da tela principal, desenvolvimento do pré-processador, desenvolvimento do processador, desenvolvimento do pós-processador do programa e análises do software obtido.

A tela inicial é o ponto de partida do programa, onde o usuário tem contato com as opções do mesmo e direciona o computador para as tarefas que deseja. $\mathrm{O}$ desenvolvimento da tela se resumiu na definição do seu lay out e na sua programação orientada objeto.

O pré-processador representa a parte do programa destinada à coleta de dados de entrada para a simulação. A elaboração do pré se iniciou com a definição do seu lay out, caracterizado pela disposição dos campos de entrada na caixa de diálogo destinados as informações inerentes à simulação e posterior programação orientada objeto.

O processador é um arquivo executável capaz de armazenar as informações provenientes do pré-processador e posteriormente executar os cálculos pertinentes à formulação definida para a análise do fenômeno desejado. Para o desenvolvimento do programa, a formulação escolhida foi a implícita e de fluxo [11]. Estabelecida a formulação, o executável foi elaborado em linguagem de programação sequencial Fortran.

O pós-processador utiliza o arquivo de resultados gerados pelo processador, para exibir na tela gráfica do programa um resultado desejado. Seguindo a mesma ideia do pré-processador, optou-se pela elaboração de um pós simples, uma caixa de diálogo disponibilizando ao usuário os resultados passíveis de exibição na tela gráfica. A etapa final do pós envolveu a programação orientada a objeto para exibição na tela gráfica do resultado selecionado na caixa de diálogo.

A análise do software obtido foi trabalhada em três etapas: escolha na literatura de simulações passíveis de serem reproduzidas; reprodução das simulações da literatura no software desenvolvido; comparação dos resultados do SALF com àqueles da literatura. A última etapa serviu de base para a análise da performance do programa obtido.

\section{RESULTADOS E DISCUSSÕES}

\subsection{Desenvolvimento da Tela inicial}

A tela inicial do programa foi definida conforme ilustrado na figura 1. Na parte superior da tela é apresentado o título do programa, SALF - Sistema de Análise de Laminação a Frio, sendo esse seguido da barra de menus. A barra é composta de dois menus: Simulação, que contém submenus do Pré-processador, Processador e Pós-processador; e Help, que contém os submenus Ajuda e Sobre. 


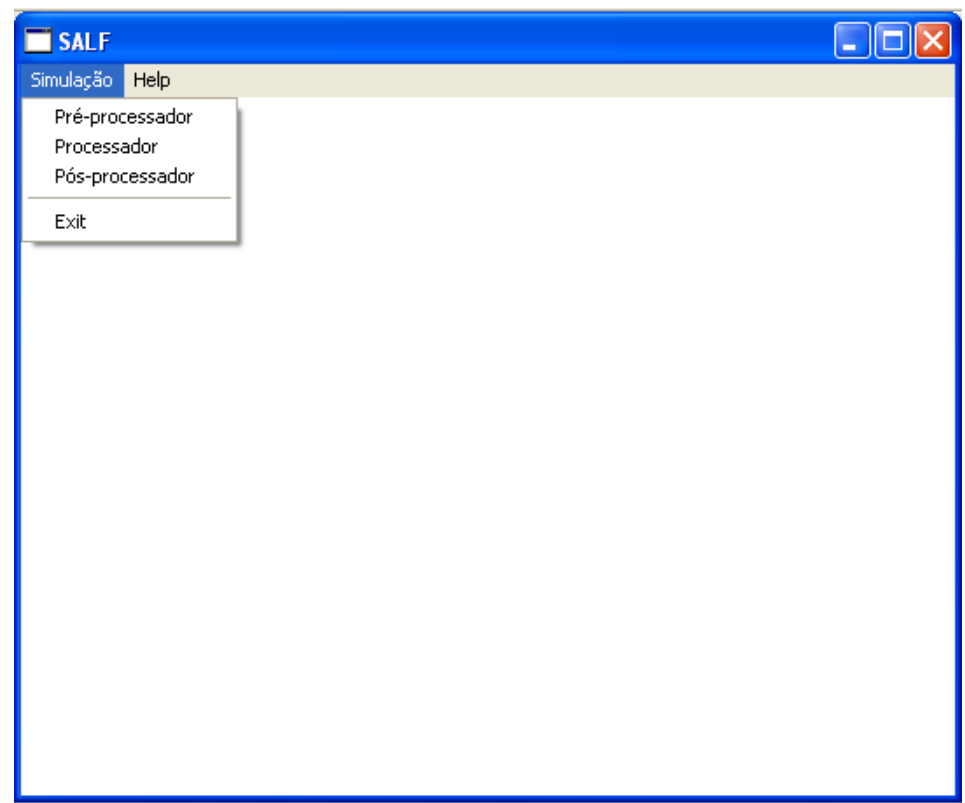

Figura 1. Tela inicial do SALF.

\subsection{Desenvolvimento do Pré-processador}

A figura 2 apresenta a caixa de diálogo caracterizando o pré-processador do programa, sendo esta exibida no momento em que o submenu Pré-processador é selecionado na tela inicial. A caixa de diálogo apresenta quatro partes, a saber: condições gerais, dados geométricos, dados do material e condições de contorno.

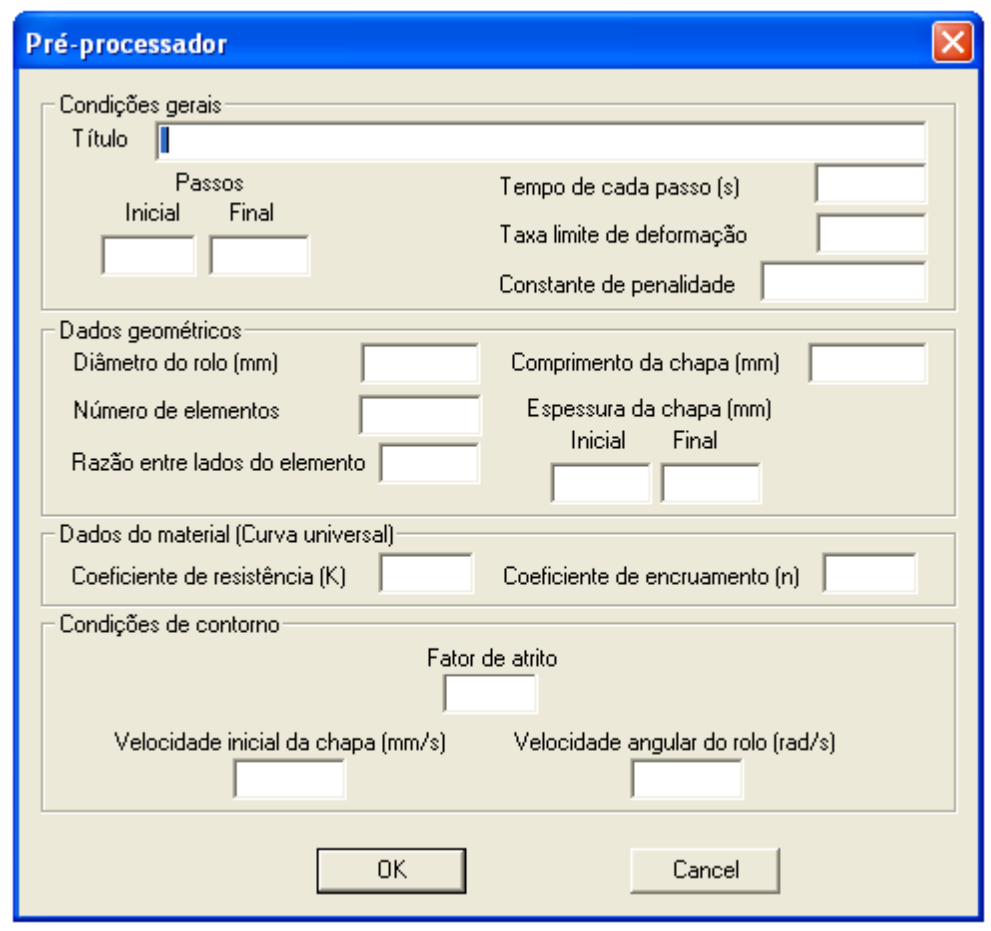

Figura 2. Pré-processador do SALF.

\subsection{Desenvolvimento do Processador}

O processador foi desenvolvido baseado na formulação implícita/rígido-plástica, seguindo a ideia básica do código de simulação do processo de recalque 
axissimétrico isotérmico fornecido na literatura [7]. As devidas adaptações no código do recalque foram feitas para a laminação a frio, sendo as principais: implementação do estado plano de deformações característico nas análises da laminação a frio; subrotina para leitura e impressão de dados de entrada e saída; desenvolvimento de uma rotina de atrito; desenvolvimento de todo o cálculo matemático para determinação da pressão de laminação, força de laminação, tensão cisalhante e torque de laminação.

\subsection{Desenvolvimento do pós-processador}

A caixa de diálogo apresentada quando da seleção do submenu Pós-processador na tela inicial é mostrada na figura 3. A parte dos Resultados Gerais na caixa apresenta os dados de saída do programa a serem exibidos na tela gráfica através da figura da chapa deformada e com a graduação de cores caracterizando a intensidade da variável analisada ao longo de toda a chapa, para o passo selecionado - ver figura 4. Na parte da caixa do Arco de Contato, os resultados exibidos estão na forma de gráficos, sendo os gráficos da pressão normal e da tensão cisalhante exibidos para cada passo e, para força e torque de laminação, o gráfico contém os resultados para todos os passos, não sendo necessário entrar com informações do passo no item seguinte da caixa (figura 5). Em Visualização, a opção passo a passo requer que o usuário selecione o passo no qual deseja visualizar o resultado de interesse.

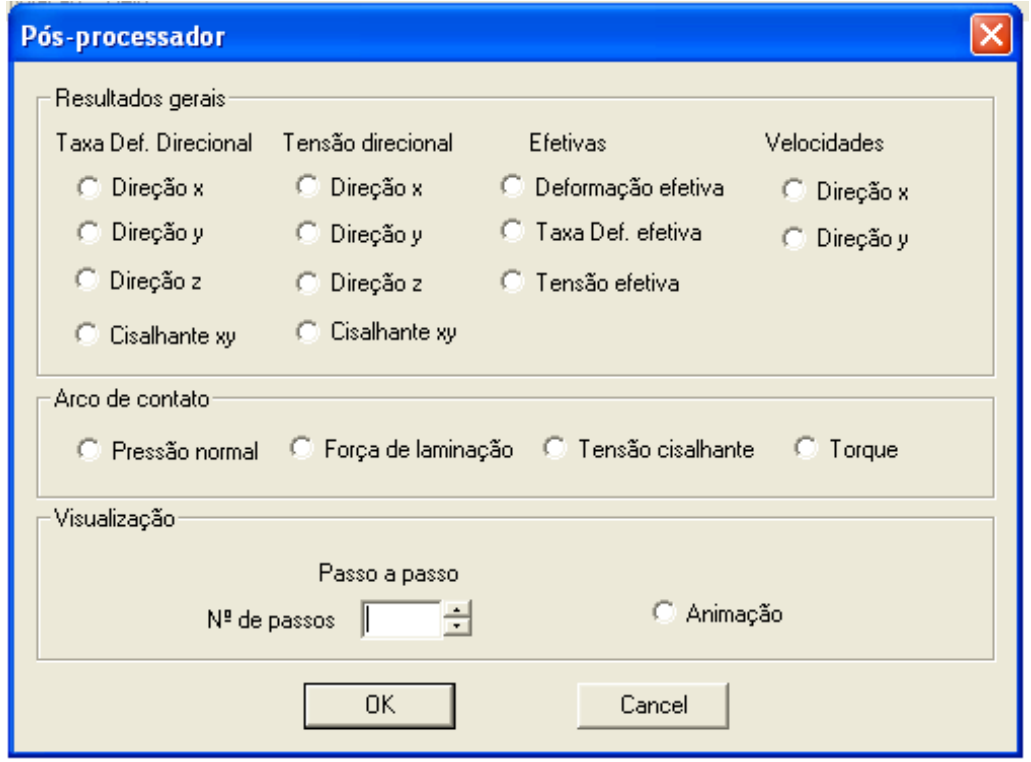

Figura 3. Caixa de diálogo do pós-processador do SALF.

Taxa de deformação efetiva

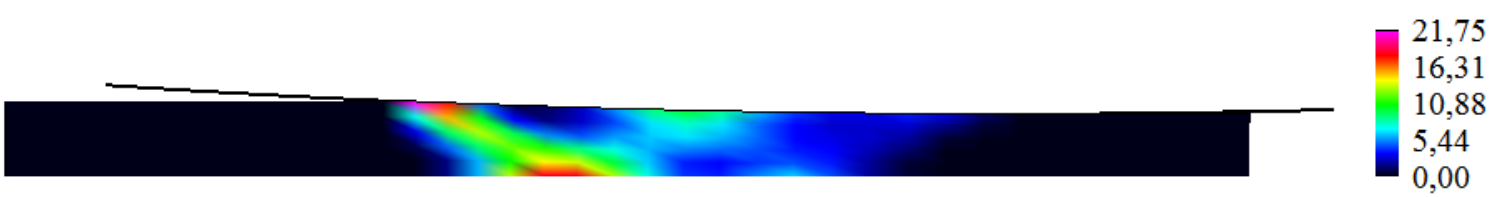

Figura 4. Ilustração de resultado obtido no item Resultados Gerais. 


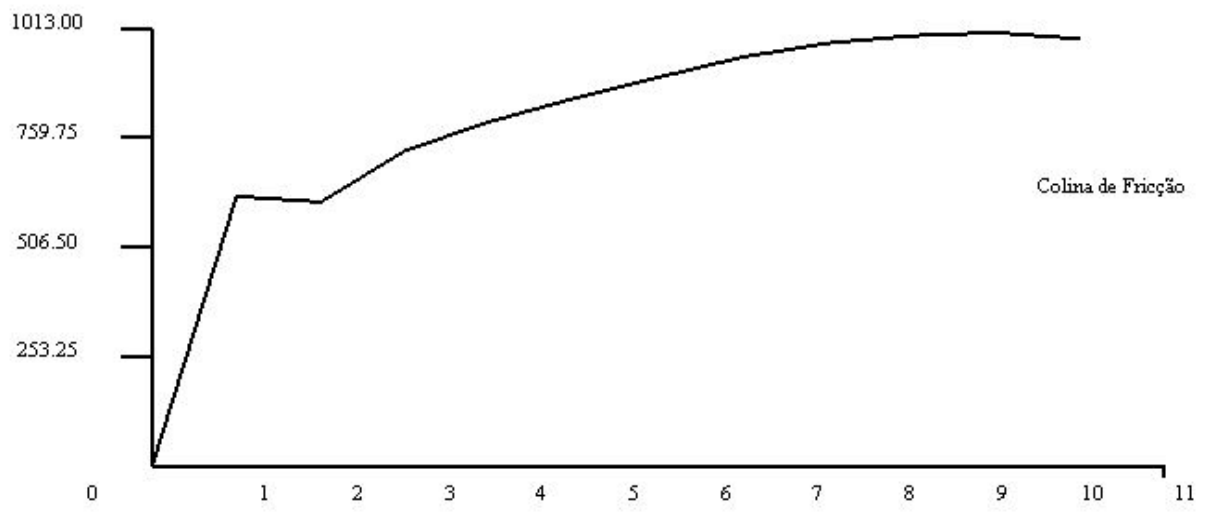

Figura 5. Ilustração de resultado obtido no item Arco de Contato.

\subsection{Simulações escolhidas}

A escolha das simulações foi direcionada de forma a buscar condições envolvendo as principais matérias primas da laminação a frio e também uma faixa de espessura representativa do processo para a força de laminação, que é um dos parâmetros mais importantes no processo de laminação a frio. Apesar do programa SALF oferecer outras opções de análise de resultados, para o trabalho em questão se focou em analisar a força de laminação, taxa de deformação efetiva e deformação efetiva. A tabela 1 apresenta as condições selecionadas para a análise da força. Para as simulações 1 e 3 também foram avaliados os resultados de taxa de deformação efetiva e deformação efetiva. Na tabela, também constam os demais parâmetros empregados nas simulações, sendo que os dados contendo asteriscos tiveram que ser arbitrados devido à falta de informação no artigo de origem.

Tabela 1. Simulações selecionadas da literatura

\begin{tabular}{cccccccc}
\hline Simulações & Material & $\begin{array}{c}\text { Espessura } \\
\text { Inicial }\end{array}$ & Redução & $\begin{array}{c}\text { Diâmetro } \\
\text { do } \\
\text { cilindro } \\
\text { (mm) }\end{array}$ & $\begin{array}{c}\text { Vel. do } \\
\text { cilindro } \\
\text { (rad/s) }\end{array}$ & $\begin{array}{c}\text { Vel. da } \\
\text { chapa } \\
\text { (mm/s) }\end{array}$ & Atrito \\
\hline $1[11]$ & Alumínio & 3,0 & $16,67 \%$ & 100 & 6,28 & 104,72 & 0,1 \\
\hline $2[11]$ & Alumínio & 3,0 & $50 \%$ & 100 & 6,28 & 104,72 & 0,1 \\
\hline $3[8]$ & Aço & 1,0 & $16 \%$ & 130 & $3,86^{*}$ & 250 & 0,06 \\
\hline $4[8]$ & Aço & 1,0 & $16 \%$ & 130 & $3,86^{*}$ & 250 & 0,15 \\
\hline
\end{tabular}

Em termos de dados de controle geral das simulações descritas na tabela 1, 0 tempo para cada intervalo, a taxa limite de deformação e a constante de penalidade foram, respectivamente, $0,001 \mathrm{~s}, 0,01 \mathrm{~s}^{-1}$ e 10000,00 . Nas simulações 1 e 2 , o número de elementos empregado na malha de cada simulação foi fornecido no artigo de origem. Para as demais simulações, o número de elementos foi definido fazendo-se uma proporção com a malha da simulação de referência.

\subsection{Análise da força de laminação}

A relação força vs redução foi analisada nas simulações 1 e 2 da tabela 1 . 0 material para estas simulações foi um alumínio com módulo de elasticidade de 69 GPa, como descrito no artigo referência de CAWTHORN et al. [11]. Considerando o módulo de elasticidade, a curva do alumínio 1100-H18 foi selecionada da literatura 
[20] para as simulações, devido a ausência desta informação no artigo fonte - ver figura 6 . A velocidade da chapa e do cilindro foram fornecidas pelo artigo de origem.

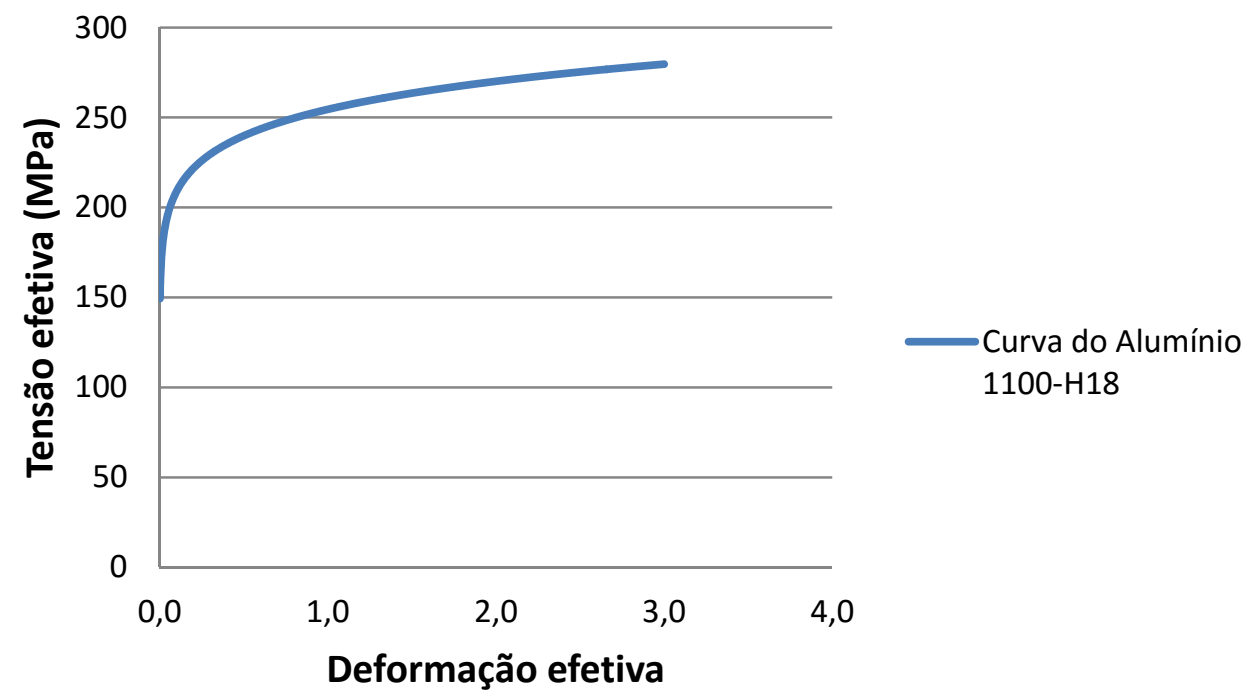

Figura 6. Curva universal usada nas simulações 1 e 2, adaptada de International Atlas of StressStrain Curves [20].

A tabela 2 apresenta os seguintes resultados: analíticos segundo o método da deformação homogênea e dos blocos; simulações no SALF; MEF de CAWTHORN et al. [11]. O SALF obteve uma melhor aproximação em relação aos resultados do artigo de origem para a simulação 2 , sobrestimando o resultado para a simulação 1 . Todos os resultados na Tabela 2 mostram que a força de laminação aumenta com o aumento da redução, conforme esperado segundo resultados da literatura [8][14][22]. A comparação dos resultados do SALF com aqueles do artigo de referência evidencia o potencial do SALF em analisar a força da laminação em função da variação da redução da espessura da chapa.

Tabela 2. Força vs redução - simulação 1: redução de 16,67\%; simulação 2: redução de $50 \%$

\begin{tabular}{cccccc}
\hline Simulação & Material & $\begin{array}{c}\text { Método da } \\
\text { Deformação } \\
\text { Homogênea }\end{array}$ & $\begin{array}{c}\text { Método } \\
\text { dos } \\
\text { Blocos }\end{array}$ & SALF & $\begin{array}{c}\text { MEF } \\
\text { CAWTHORN, } \\
\text { et al [11] }\end{array}$ \\
\hline $1(\mathrm{kN})$ & Alumínio & 31,8 & 39,3 & 27,4 & 20 \\
\hline $2(\mathrm{kN})$ & Alumínio & 59,2 & 69,5 & 50,6 & 50 \\
\hline
\end{tabular}

Nas simulações 3 e 4 , o artigo de origem fornece a curva universal do aço utilizado para a análise $\left(\sigma=324(1+19,23 \bar{\varepsilon})^{0.295} \mathrm{MPa}\right)$. As velocidades da chapa e do cilindro não foram fornecidas pelo artigo em questão, sendo então arbitradas, conforme explicado anteriormente.

A tabela 3 apresenta os resultados dos métodos analíticos, do SALF, do MEF e experimentais de PRAKASH, et al. [8]. De acordo com os dados do artigo, a força de laminação permanece quase constante com a variação do atrito. $O$ mesmo comportamento pode ser percebido nos resultados do SALF e no método da deformação homogênea. A constância da força com a variação do atrito também é vista em CAWTHORN et al. [11]. Em termos quantitativos, o resultado experimental de PRAKASH, et al. [8] e do SALF ficaram próximos, enquanto o resultado numérico de PRAKASH, et al. [8] ficou distante do seu resultado experimental. Desta forma 
nota-se claramente que o SALF, em princípio, consegue reproduzir a relação força vs atrito corretamente.

Tabela 3. Força vs atrito - simulação 3: atrito de 0,06; simulação 2: atrito de 0,1

\begin{tabular}{ccccccc}
\hline Simulação & Material & $\begin{array}{c}\text { Método da } \\
\text { Deformação } \\
\text { Homogênea }\end{array}$ & $\begin{array}{c}\text { Método } \\
\text { dos } \\
\text { Blocos }\end{array}$ & SALF & $\begin{array}{c}\text { MEF } \\
\text { PRAKASH, } \\
\text { et al [8] }\end{array}$ & $\begin{array}{c}\text { Experimental } \\
\text { PRAKASH, } \\
\text { et al [8] }\end{array}$ \\
\hline $3(\mathrm{kN} / \mathrm{mm})$ & Aço & 1535 & 2213 & 3276 & 1900 & 3200 \\
\hline $4(\mathrm{kN} / \mathrm{mm})$ & Aço & 1535 & 2758 & 3305 & 1950 & - \\
\hline
\end{tabular}

\subsection{Análise da taxa de deformação efetiva e da deformação efetiva}

As figuras neste subitem mostram os resultados do programa SALF para a taxa de deformação efetiva e a deformação efetiva para as simulações 1 e 3, com objetivo de avaliar o comportamento do programa quanto a essas variáveis.

A plotagem da taxa de deformação efetiva é apresentada nas figuras 7 e 8 . Os dados obtidos estão de acordo com a realidade do processo, ou seja, a taxa de deformação efetiva tende a aumentar à medida que o material caminha para dentro do arco de contato e, quando o material começa a deixar a região do arco de contato, a taxa tem que se aproximar de zero. Resultados similares são obtidos por e LIN e SHEN [21] e por KOMORI [22].

Taxa de deformação efetiva

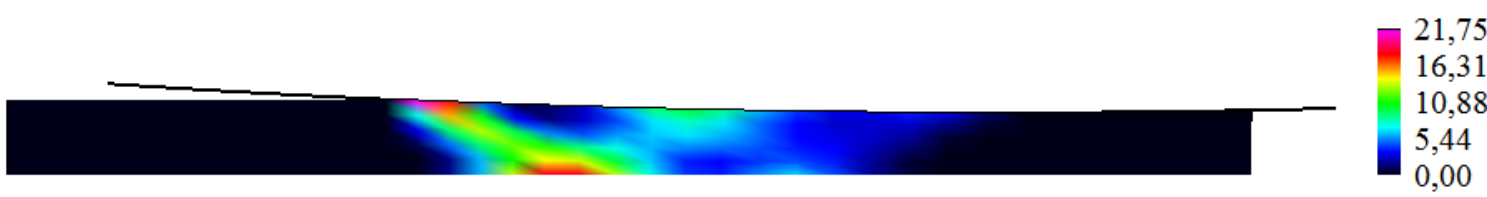

Figura 7. Taxa de deformação efetiva - Espessura de $3 \mathrm{~mm}$, redução de 16,67\%, velocidade da chapa de $104,72 \mathrm{~mm} / \mathrm{s}$ (simulação 1$)$.

Taxa de deformação efetiva

$\begin{array}{r}\text { Taxa de deformação efetiva } \\ \\ \hline \\ \hline \\ \hline\end{array}$

Figura 8. Taxa de deformação efetiva - Espessura de $1 \mathrm{~mm}$, redução de 16\%, velocidade da chapa de $250 \mathrm{~mm} / \mathrm{s}$ (simulação 3).

As figuras 9 e 10 apresentam os resultados da deformação efetiva. Nota-se que os maiores valores de deformação efetiva são observados nas regiões onde as taxas de deformação efetiva são altas. Em ambas as espessuras analisadas, percebe-se que a deformação efetiva tende a aumentar da entrada da chapa para a sua saída, seguindo o comportamento já conhecido experimentalmente. $O$ efeito da pressão do cilindro sobre a chapa tende a diminuir ao longo de sua espessura, à medida que a altura inicial da chapa aumenta, conforme pode ser visto na figura 9. Esse fato certamente dependerá da intensidade da redução da chapa, ou seja, quanto maior a redução maior será o efeito da pressão contribuindo para elevar o valor de deformação efetiva ao centro da chapa, fato este corroborado pelos resultados obtidos por RICHELSEN [23]. 


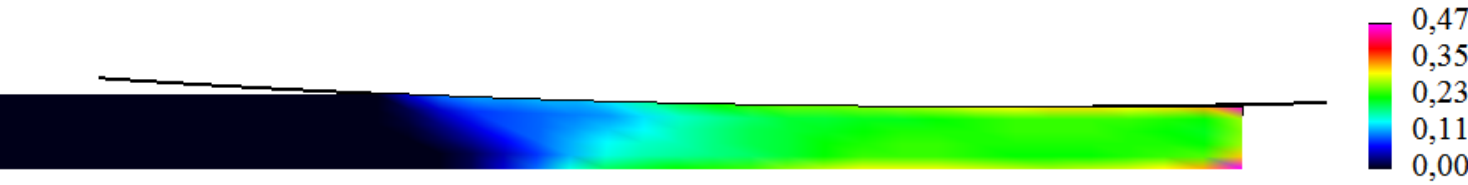

Figura 9. Deformação efetiva - Espessura de 3 mm, redução de 16,67\% (simulação 2).

Deformação efetiva acumulada

Figura 10. Deformação efetiva - Espessura de $1 \mathrm{~mm}$, redução de $16 \%$ (simulação 3).

\section{CONCLUSÃO}

De acordo com os resultados do item anterior, o programa SALF mostrou estar apto para simular o processo de laminação a frio. Sendo assim, a partir de agora, o setor siderúrgico brasileiro tem uma ferramenta nacional capaz de simular seus processos através do MEF. Os passos seguintes para evolução do programa consistem em simular outras condições com dados obtidos da indústria. Estas simulações irão fornecer informações sobre o desempenho do programa dentro do range de condições na laminação a frio, mostrando então os ajustes necessários ao programa de acordo com a demanda da siderurgia.

Além disso, outras ferramentas serão acopladas ao SALF futuramente, sendo estas: incorporação de um modelo para análise da transferência de calor, que possibilitará análise termomecânica, expandindo assim a capacidade do programa para análises a quente; desenvolvimento de modelo de dano durante o processo, permitindo ao programa analisar possibilidade de danos no material durante a laminação. Desta forma, o SALF será capaz de cobrir todo o universo do processo de laminação.

\section{REFERÊNCIAS}

1 SICETEL. Sindicato Nacional das Indústrias de Trefilação e Laminação de Metais Ferrosos. Disponível em: <http://www.sicetel.org.br/>. Acessado em: Feb 29, 2016.

2 THOMSEN, E. G.; CHARLES, T. Y.; KOBAYASHI, O. S. Plastic Deformation in Metal Processing. $1^{a}$ Edição. ed. [S.I.]: Macmillan, 1965.

3 AVITZUR, B.; CHOI, J. C.; KIM, B. M. Upper-bound solutions for several metal-forming processes. NAMRC XIV. [S.I.]: [s.n.]. 1986. p. 406-413.

4 HOSFORD, W. F.; CADDELL, R. M. Metal Forming - Mechanics and Metallurgy. $2^{\mathrm{a}}$ Edição. ed. [S.I.]: Prentice Hall PTR, 1993.

5 BRESCIANI, F. et al. Conformação Plástica dos Metais. 5a Edição. ed. Campinas: Unicamp, 1997.

6 HELMAN, H.; CETLIN, P. R. Fundamentos da Conformação Mecânica dos Metais. $2^{a}$ Edição. ed. Belo Horizonte: Artliber, 2005. 
7 KOBAYASHI, O. S.; ALTAN, T. Metal Forming and Finite Element Method. $1^{\text {a }}$ Edição. ed. New York: Oxford University Press, 1989.

8 PRAKASH, R. S.; DIXIT, P. M.; LAL, G. K. Steady-state plane-strain cold rolling of a strain-hardening material. Journal of Materials Processing Technology, 52, 1995. 338358.

9 ABO-ELKHIER, M. Elasto-plastic finite element modelling of strip cold rolling using Eulerian fixed mesh technique. Finite Elements in Analysis and Design, 27, 1997. 323334.

10 JIANG, Z. Y.; WEI, D.; TIEU, A. K. Analysis of cold rolling of ultra thin strip. Journal of Materials Processing Technology, 209, 2009. 4584-4589.

11 CAWTHORN, C. J.; LOUKAIDES, E. G.; ALLWOOD, J. M. Comparison of analytical models for sheet rolling. Procedia Engineering, 81, 2014. 2451-2456.

12 CHEN, J. et al. Void closure prediction in cold rolling using finite element analysis and neural network. Journal of Materials Processing Technology, 211, n. 2, 2011. 245-255.

13 MASHAYEKHI, M.; TORABIAN, N.; POURSINA, M. Continuum damage mechanics analysis of strip tearing in a tandem cold rolling process. Simulation Modelling Practice and Theory, 19, n. 2, 2011.

14 CETLIN, P. R.; MESQUITA, E. L. A. Plasticina na análise de deformação na laminação. Metalurgia ABM, v. 35, p. 829-832, 1979.

15 THOMSON, P. F.; BROWN, J. H. A study of deformation during cold rolling using visioplasticity. International Journal of Mechanical Sciences, 24, n. 9, 1982. 559-576.

16 CETLIN, P. R.; LISBOA, R. S. M.; GARCIA, F. Otimização da Laminação de Desbaste Através da Simulação Física Utilizando Plasticina. XXXII Seminário de Laminação de Processos e Produtos Laminados e Revestidos. Curitiba: Ed. ABM. 1995. p. 597-608.

17 CETLIN, P. R. et al. Emprego da Plasticina na determinação do coeficiente de atrito na conformação mecânica. XXXIV Seminário de Laminação - Processos e Produtos Revestidos e Laminados. Belo Horizonte: Ed. ABM. 1997. p. 331-340.

18 ESCRIBANO, R. et al. Modelling a Skin-Pass Rolling Process by Means of Data Mining Techniques and Finite Element Method. International Journal of Iron and Steel Research, 19, n. 5, 2012. 43-49.

19 RAMBERG, W.; OSGOOD, W. R. Description of Stress-Strain Curves by Three Parameters. National Advisory Committee for Aeronautics, Washington, 1943.

20 ATLAS OF STRESS-STRAIN CURVES, $2^{\text {a }}$ Edição. United States of America. ASM International, 2004.

21 LIN, Z.; SHEN, C. A rolling process two-dimensional finite element model analysis. Finite Elements in Analysis and Design, 26, 1997. 143-160.

22 KOMORI, K. Rigid-plastic Finite Element Method for analysis of three-dimensional rolling that requires small memory capacity. International Journal of Mechanical Sciences, 40, 1998. 479-491.

23 RICHELSEN, A. B. Elastic-plastic Analysis of the stress and strain distribution in asymmetric rolling. International Journal of Mechanical Sciences, 39, 1997. 1199-1211 